\title{
Cartographie de la nouvelle québécoise contemporaine
}

\author{
Gaëtan Brulotte \\ University of South Florida
}

$L$ e genre de la nouvelle s'est considérablement au Québec au cours du dernier recueils dans lesquels les auteurs rivalisent d'ingéniosité dans leur recherche de la qualité et où l'audace formelle côtoie la profondeur. Nul doute, la nouvelle est le genre littéraire idéal pour expérimenter sur l'art narratif, mais est-elle aussi innovatrice dans ses considérations socio-culturelles? Pour le savoir, je vais me pencher sur un aspect crucial dans ce domaine, les représentations de l'espace et de la sexuation? Pour explorer cet aspect j'ai fondé mes analyses sur un échantillon de quatre nouvellistes vivants, deux femmes, deux hommes. Il s'agit de Aude, Élise Turcotte, Bertrand Bergeron et Jean Pierre Girard. Ces auteurs me semblent assez représentatifs des orientations récentes du genre et ces quatre œuvres, qui sont d'ailleurs de longueur comparable, offrent un eventail proche d'une soixantaine d'histoires parues au cours des dix dernières années, de 1993 à 1997. Au-delà des critères esthétiques qui ont été déterminants dans ma sélection (ce sont des recueils d'une bonne tenue littéraire), ces auteurs ont en commun, à l'instar de la plupart des nouvellistes québécois de la dernière décennie, de représenter dans leurs œuvres fictives surtout des relations hétérosexuelles. A cet égard il m'est apparu intéressant, pour l'analyse de la sexuation spatiale, de voir justement comment ils mettent en scène les deux sexes dans leurs rapports à l'espace. Vu l'angle d'attaque de ce travail le critère spatial a bien évidemment compté dans mon choix, ces œuvres offrant des mises en espace riches et diversifiées. Le titre de ces œuvres propose déjà tout un programme territorial: Espaces à occuper avec ses connotations de découverte et de conquête, Caravane avec ses suggestions de parcours, Visa pour le réel et ses évocations frontalières, ses expériences perceptives de l'espace, Cet imperceptible mouvement ne fût-ce que pour le paramètre si important de la mobilité et de l'immobilité. En outre je n'ai pas négligé la représentation géographique, car dans ma sélection, il y a une nouvelliste de Montréal, une autre de Québec et deux de différentes régions de la Province, la Mauricie et la Beauce, ne serait-ce que pour vérifier si le traitement de l'espace est influencé par l'origine géographique de l'écriture.

Pour l'analyse j'ai tout simplement soumis ces quatre oeuvres à la même grille de paramètres spatiaux, soit public/privé, ville/campagne, fermeture/ouverture, mobilité/immobilité et les entre-deux symboliques, parametres qui constituent les divisions de mon propos. 


\section{Public/privé}

Les paramètres public/privé sont les premiers qui sautent aux yeux dans la nouvelle québécoise contemporaine. Après d'autres dont Richard Sennett, Nancy Duncan affirme dans Bodyspace, ouvrage collectif qu'elle a dirigé en 1996, que cette distinction est désormais motivée par le genre et a pour but implicite de réguler la sexualité (128). Pour elle le privé est traditionnellement (et notamment) associé au domestique, au naturel, au corporel, à la famille, à la propriété, au foyer, à la vie personnelle, à l'intimité, à la passion sexuelle, aux soins, au port d'attache, au travail non rémuneré, à la reproduction et à l'immanence. Le public, lui, est le domaine du désincarné, de l'abstrait, du culturel, de la rationalité, du discours critique, de la citoyenneté, de la cité, du politique, de la société civile, de la justice, du marché, du travail rémunéré, de la production, de l'action, du militarisme, de l'héroïsme, de la transcendance. Ces espaces sont déterminés selon le genre sexuel et sont rigoureusement hétérogènes à ses yeux: le privé est féminin et le public, masculin, les hommes ayant toujours pu se déplacer de l'espace privé à l'espace public avec plus de fluidité, de légitimité et de sécurité que les femmes.

Voyons donc comment la nouvelle québécoise actuelle traite cette distinction reçue. Les nouvellistes ménagent à l'envi pour les deux sexes des espaces minimaux où échanger et interagir. Les auteurs, féminins et masculins, nous y montrent abondance de héros et d'héroïnes qui vivent l'évasion dans des espaces publics tels que bars, boîtes ou cafés. La Marie de Turcotte, par exemple, aime sortir seule pour assister à un spectacle de jazz, prendre un verre, s'enivrer de musique ou danser seule ("Ligne de tir"). Le lieu public sert de tremplin à la recherche d'un réconfort, à la jouissance de moments d'intensité, mais aussi à la quête de nouvelles formes d'intimité entre les sexes (voyez "L'écoute active", "La virtuelle" ou "Soirée barattée" de Girard, par exemple), même si ces rencontres ne débouchent souvent que sur la chasteté ou l'exercice d'une sexualité primaire et expéditive où le bonheur sensuel n'est guère au rendez-vous.

Dans la nouvelle contemporaine, l'espace public n'est cependant pas qu'un domaine d'évasion et de rencontres étourdies pour l'un ou l'autre sexe, ce qui est devenu tout compte fait banal. Plus profondément ces lieux apparaissent comme des lieux de transcendance féminine et d'aliénation masculine. Pour Turcotte le métro surtout est le lieu révélateur de soi à travers la prise de conscience du tragique des autres et de l'absurdité de la vie. Son hérö̈ne rapporte de son bain de foule des visions bouleversantes qui lui plantent un couteau dans le cœur, puisqu'elle y découvre une vitrine de la misère humaine et cette misère atteint les deux genres. Elle voit notamment un garçon aliéné qui frappe dans la vitre du métro sans raison avant de se rasseoir calmement sur son banc. "Je l'ai vu faire, dit-elle dans "Le paradis de tous les possibles", et je suis sortie du wagon avec une partie de ce geste et de ces yeux bleus bien tapie en moi. La vie me permet parfois d'oublier. Pas cette fois." (83) Elle observe encore une vieille dame immobilisée devant un escalier roulant, incapable de se décider à l'emprunter: le lendemain, chavirée par cette scène, Marie aura du mal à son tour à mettre

\section{LittéRéalité 60}


le pied sur la première marche ("Caravane", 33). Et qui est-ce encore qui la frappe dans le métro? Un homme dessinant de lignes erratiques le portrait des passagers; une voisine de banquette lisant un livre sur "les choses que je ne suis pas capable de dire"; une femme nerveuse, obsessive étalant ses sacs de courses en public et qui importune tout le monde. A quoi s'ajoutent un jeune homme qu'elle cherche à séduire mais qui regarde sa montre et un homme âgé qui l'aborde en s'écrasant d'une crise cardiaque à ses pieds. "J'étais tous ces gens" conclut Marie (39) qui sent alors une transformation majeure s'opérer en elle, car elle décide subitement de quitter son emploi, décision totalement libératrice. "Je sentais un surplus d'énergie s'évader de mon corps. Je sentais ce souffle remonter en moi puis s'évader pendant que la vie entrait." (39) La misère des autres agit donc comme un fouet sur elle et se métamorphose en vitalité nouvelle. L'espace public peut devenir un espace troublant, certes, mais il soulève aussi une remise en question profonde et une redéfinition des valeurs. Ici l'héroïne réussit à dépasser la tragédie humaine sur laquelle elle ouvre les yeux pour la subsumer dans une transcendance qui l'incite à changer ses orientations de vie.

Par opposition, l'espace public pour le genre masculin sera le lieu de l'aliénation où se concentrent les obligations formelles et socio-économiques. Cet aspect s'illustre à merveille chez Girard, et nulle part mieux que quand le nouvelliste représente des lieux de travail dans deux nouvelles "L'élue" et "L'effigie oubliée". Dans ces deux cas l'espace laborieux est un monde inhumain habité par la contrainte, la tension, la violence, la bêtise et d'où aucun épanouissement ne peut transpirer. Dans "L'élue", en une fable moderne très sombre, Girard montre l'univers insoutenable d'un poulailler à son moment de transition quand on le vide de ses locataires pour faire place nette aux prochains poussins. L'auteur adopte pour une partie de l'histoire le point de vue d'une poule de cette couvée qui est destinée à la consommation. C'est un espace de brutalité musclée où on saisit les animaux sans ménagement par les pattes, les met en cage, les charge dans un camion qui les emporte en Ontario. La poule "privilégiée" (si l'on peut dire) de cette nouvelle trouvera la mort sur l'autoroute quand un barreau de sa cage se rompra. Elle n'aura ainsi connu qu'un très bref moment de liberté, purement accidentel. Or en parallèle à cette tragédie animale, Girard s'intéresse aussi au sort du producteur de ce poulailler et qui n'est guère mieux tout compte fait: c'est un vieil homme primaire écrasé par la vie qui fait ses besoins sur place dans un coin et qui est étourdi par l'alcool, ce qui lui permet sans doute de soutenir l'insoutenable. Il est abruti par un système économique qui le dépasse et aux chaînes duquel il est totalement soumis. Les lampées de gin l'aident, certes, à engourdir sa conscience, mais l'empêchent assurément de se poser des questions comme le fait la Marie de Turcotte. Il est aliéné dans son labeur solitaire, condamné à la répétition des gestes et à la reproduction du systeme à l'homéostasie duquel il participe. Ici aucune transcendance n'est possible. Cet anti-héros est enlisé dans l'immanence, dans une existence au ras du fumier, vouée à l'éternel retour, faite de service et de sacrifice pour la collectivité.

C'est un univers tout aussi étouffant que "L'effigie oubliée" offre, en moins industriel cette fois, puisque le héros est artisan menuisier et son espace public se réduit à son atelier. 
Ici l'ouvrier subira l'agression d'un de ses clients qui le conduira à la mort. A l'origine de l'affaire, deux facteurs que Girard fait ressortir: d'une part, la maladresse du menuisier qui, par accident, casse un meuble à la restauration duquel il œuvrait pour son client; et d'autre part, l'état d'esprit particulier du client, alors sous le choc d'une scène de ménage, ce qui le montre victime de ces tyrannies de l'intimité dont a pu parler Sennett (1974). Les tensions du privé se traduisant en acting out public maladroit et aggravant, il deviendra agresseur à son tour, voire meurtrier involontaire. Le tout est raconté dans la langue aliénée du joual, et c'est la seule du recueil à adopter cette forme stylistique, ce qui renforce le caractère primaire des hommes en cause et le sentiment d'oppression qui domine leur univers public.

Avec Girard nous avons une étude de l'espace public au masculin qui est un espace d'aliénation, d'emprisonnement, d'étouffement, de violence, de malheur, aliénation sur laquelle se repose le reste de la société pour survivre et jouir de ses vastes loisirs.

Le traitement le plus spectaculaire de l'espace public vient cependant de la déterritorialisation du sexuel par les nouvellistes. La sexualité de l'homme et de la femme n'est plus confinée entre quatre murs dans le confort d'un lieu privé, elle éclate au grand jour dans des endroits publics. Turcotte nous présente des amants s'embrassant contre un mur d'un centre commercial ou s'unissant sur le billard électrique d'une "arcade" (130), ou encore dans un escalier, des toilettes publiques, etc. "Ça se passait n'importe où", ajoute-t-elle (139). Girard, lui, choisit la grande ville de Paris pour y mettre en scène la naissance de l'amour entre un homme et une femme qui exhibent insolemment leur sexualité à la boutonnière, ce qui gêne les autres. Ils déambulent tous les deux, lui en lui couvrant un sein de la main et elle, en lui glissant ses doigts dans la poche de son jean. Les passants désapprouvent nettement cet exhibitionnisme hétérosexuel et le narrateur réagit à ce rejet par un rejet plus prononcé encore: "Les passants me dévisagent", "Je hais tous ceux qui se retournent", "J'encule les passants" (173). L'exercice de la sexualité devient consciemment transgressive et provocatrice.

Dans ces deux cas, Turcotte et Girard, les nouvellistes mettent en scène un conflit entre la sexualité, si minimes qu'en soient les signes extérieurs, et un espace public perçu comme tacitement asexué. Tout affichage de sexualité devient choquant dans un lieu où, par convention, le corps devrait s'efforcer de s'absenter. En transgressant cette règle, ces auteurs témoignent aussi d'un désir de transformer les données spatiales qui sont ressenties comme répressives et de briser les frontières trop étanches entre le public et le privé.

Recoupant les paramètres du public, la mobilité des personnages des deux sexes vient renforcer cette déterritorialisation de la sexualité. D'une manière frappante la voiture devient chez beaucoup d'entre eux un lieu d'intimité érotique. Chez Aude on s'y embrasse en sortant de l'école, ce qui serait banal aujourd'hui si ce n'était que les deux personnages amoureux sont oncle et nièce, qu'elle est mineure et qu'il a le double de son âge ("Iris"). Le geste cumule discrètement une multitude de transgressions. Chez Turcotte on s'y caresse ("C'est dans les voitures qu'on se touche le plus" fait-elle dire à Marie (141)), l'homme y déshabille sa partenaire, la femme y jouit tout son content. Autant cependant Turcotte

\section{LittéRéalite 62}


mentionne toujours le plaisir féminin et jamais le masculin, comme si la femme éprouvait et l'homme n'était que l'instrument qui faisait éprouver, autant Girard est plus égalitaire dans "La Femme-Subaru" où il accorde à tour de rôle un orgasme démocratique à ses deux personnages, la conductrice et son passager auto-stoppeur, plaisir routier qu'ils se donnent tout en roulant à 140 à l'heure. J'ai déjà baptisé Girard le grand coureur automobile de la nouvelle québécoise contemporaine tant la voiture chez lui est un espace privilégié pour

véhiculer la grande mobilité du désir. Plusieurs de ses nouvelles se passent sur quatre roues ${ }^{2}$. "343 Nord" tient tout entière dans le parcours désirant du narrateur au volant d'un camion qui convoite une jeune fille assise au fond d'un bus. Dans "Lidylle" un automobiliste initie une drague à haute vitesse avec une conductrice qui bientôt renchérit sur lui. Le désir ne se limite plus aux refuges privés culturellement réservés à son expression: la route devient un espace de désir où la sexualité s'affirme, ne fût-ce que dans l'éphémère. Girard est bien conscient lui aussi du caractère transgressif de cette déterritorialisation puisque son héros se fait finalement interpeller par la police, la loi venant ainsi remettre temporairement de l'ordre dans la pagaille des territoires.

En conclusion, l'espace public inverse ici les associations traditionnelles: pour l'homme il n'offre plus l'epanouissement qu'on lui attribuait naguère et n'est d'aucun secours au malaise existentiel. Il condamne les conduites masculines à la répétition, à l'emprisonnement, à l'étouffement, à l'immanence. Dans les interactions entre les sexes qu'il permet le lieu public ne débouche guère que sur un érotisme passager d'où rien de durable ne sort. Autant pour l'homme il n'est plus lié à la liberté comme il l'a traditionnellement été, autant pour la femme il n'est plus associé à la disgrâce ainsi qu'il l'a longtemps été. Il constitue plutôt pour elle un lieu d'évasion où déverser un trop plein d'angoisse et peut même devenir une sphère de transcendance si on sait intérieurement se l'approprier. La polarisation générique inversée de l'espace public apparaît plus vive encore dans le traitement du travail: la femme le quitte facilement pour connaître des joies libératrices immédiates, alors que l'homme s'y sent piégé, prisonnier, aliéne, aucune lucidité ne venant l'éclairer, aucune distance critique ne lui permettant de respirer. Pour les deux sexes cependant, il peut constituer une source de fortes intensités, surtout si on laisse les audaces spatiales du désir faire éclater les frontières du privé. La sexualité est alors vécue sur un mode public comme un défi aux conventions et se donne comme la seule soupape déterritorialisante efficace. Le désir se met en route littéralement, l'intimité cherche à se vivre dehors et n'est plus parquée chez soi.

\section{Le privé}

Reste le privé dont on pourrait penser qu'il peut constituer un refuge. Voyons de plus près ce qu'il en est.

Chez Turcotte l'espace privé c'est l'appartement, lieu d'une petite famille monoparentale où Marie vit avec ses deux enfants et occasionnellement son ami (mentionné dans "Une tache"). On y voit l'hérö̈ne dans ses activités domestiques, lesquelles se présentent 
sur un mode en général heureux: rangement, récurage, toilette personnelle (25). D'une nouvelle à l'autre, on l'y voit cuisiner, célébrer Noël avec ses enfants, recevoir ses amies féminines, y échanger autour d'un porto ou d'une bière, y accueillir un amant de passage, interagir avec les voisins et simplement décompresser seule devant un verre. Cependant cet espace privé est sans cesse menacé de l'extérieur et envahi par le public de sorte qu'il se montre fragile au point de perdre ses attributs d'un chez soi protecteur. Dans "Le paradis de tous les possibles" l'héroïne vit une obsession révélatrice: elle a sans cesse peur que sa porte soit mal fermée, elle rêve la nuit que son domicile est cambriolé. Dans "Enfance", Turcotte semble donner une clé personnelle à cette foncière fragilité territoriale quand elle évoque les fréquents déménagements familiaux, conséquence des mariages qui se défont, et qui ont créé chez elle un sentiment de déracinement perpétuel. Dans un des nouveaux logements où elle a échoué, elle observe que les lumières étaient sans cesse éteintes et, y voyant un signe, elle constate que "toujours il y avait une menace" (104). Plus tard l'intimité sera, par conséquent, difficile à éprouver dans sa plénitude, ne serait-ce qu'à cause des voisins qui la gênent, soit parce qu'elle se sent observée par la fenêtre, soit par les bruits qui lui parviennent et leurs drames domestiques étalés au grand jour. Par exemple dans "La robe de mariée", Françoise, la voisine d'en face, sort dehors sur le perron devant la maison pour pleurer, pour qu'on la voie souffrir. La narratrice elle-même peut entendre une autre femme habitant tout près chanter et peut l'observer dans ses activités quotidiennes les plus personnels (comme parler au téléphone, se brosser les cheveux, danser dans le salon, préparer les repas, battre les enfants), comme elle épie un voisin qui écoute de la musique à plein volume đans sa voiture (notons encore qu'un espace privé vient élire domicile dehors).

On observe donc une sorte de brouillage des espaces privés et publics, les deux interférant sans cesse. Ce brouillage territorial apparaît encore dans la façon dont se vit l'intimité à deux chez une femme comme Turcotte. Quand son hérö̈ne se retrouve dans le même espace qu'un homme, elle est plus à l'aise dans une situation sociale de séduction que dans une situation de forte proximité. C'est ce qui se passe dans "Comment vivre le soir de son anniversaire" où elle décide de charmer un inconnu à l'occasion d'une partie chez des amis. Dans ce lieu semi-public rempli d'invités elle se montre entreprenante auprès de cet homme, l'approche explicitement en lui mettant sa main sous le chandail, boit dans son verre, l'entraîne vers la bibliothèque où ils s'embrassent, a des gestes passionnés envers lui et finalement colle sa tête sur son ventre. Cependant sitôt arrivée chez elle avec lui en territoire familier elle vit son intimité dans l'embarras, maintenant une froide distance entre eux. Elle s'agite sans arrêt, passant son temps à apparaître et à disparaître avant de lui faire voir finalement sa vidéo favorite qui met en scène un oiseau de proie en train de manger sa victime. Ils regardent ce court metrage cruel ensemble, mais séparés, lui couché, elle assise par terre, chacun dans son micro espace. Elle le lui repasse plusieurs fois, car elle veut lui faire mal. Au matin il comprend qu'il doit s'en aller et rien d'autre ne se passera entre eux. La plus grande des intimités, la conduite sexuelle, est apparemment heureuse en public, mais impossible en privé, là où elle devait s'épanouir dans les conceptions territoriales reçues.

\section{LittéRéalité * 64}


Aude est la seule à montrer le bonheur d'un espace domestique partagé par les deux sexes, mais il est le fruit d'une adaptation réciproque. Dans "Fleurs de pavot", le héros, qui est écrivain, a loué une maison de campagne pour s'isoler afin de mener à terme un projet. Sa compagne Florence, qui est artiste, l'y a suivi. Les deux cuvrent au rez-de-chaussée dans une seule grande pièce ouverte où se juxtaposent les aires de la cuisine, du séjour, de la salle à manger, de la salle de bains et leur coin de travail respectif. Pour la première fois, ils se trouvent à partager le même espace de travail. Au début il en est incommodé, puis finit par redécouvrir sa compagne dans ses activités quotidiennes alors qu'il croyait tout savoir sur elle. Un changement d'espace, une mise en commun temporaire suffisent à revitaliser une relation. Chez Aude l'espace privé partagé à deux représente même la condition de base pour le bonheur de ses personnages, car idéalement il est toujours gémellaire. Quand il n'est plus partagé il se dévalue et se transforme en lieu d'accablement et de solitude souffrante, ce qui survient suprêmement dans les situations de maladie ou d'agonie à l'hôpital où la destruction du privé par le public, autant pour l'homme que pour la femme, ne peut renvoyer qu'à la mort.

Voilà bien ce qu'elle montre dans deux de ses nouvelles, "Vases communicants" et "Tout est ici", qui sont les plus troublantes de toute la dernière décennie. Dans "Tout est ici" le héros narrateur est malade et cloué au lit dans un asile où tout est sale, froid, glacial, sinistre. Il n'y a, dans ce lieu infâme, ni table de nuit, ni bureau, ni chaises, ni espaces de rangement pour les effets personnels. Rien, sauf un lit, "le seul endroit, dit-il, qui soit vraiment chez moi" (12). Ici tout le privé disparaît au profit d'un espace public écrasant. De cet enfer médical, il n'y a aucun espoir de sortir un jour. "Vases communicants" est le pendant féminin de "Tout est ici" et nous y voyons cette fois une hérö̈ne agonisant au milieu de douze autres lits dans un hôpital que l'on ne quitte que les pieds devant. La maladie ou l'agonie suppriment radicalement l'intimité avec l'autre, confinent l'être à la solitude et abrogent le droit à l'espace personnel. En fait, chez Aude la destruction du privé ne peut signifier que la mort imminente, tant l'espace personnel est vital dans son univers.

Chez les héros masculins l'espace privé est davantage assailli de toutes parts et semble impossible à protéger. C'est tout à fait frappant chez Bergeron où il est sans cesse agressé et violé. On le voit chez un de ses héros surpris au retour du travail de découvrir qu'on a repeint son intérieur ou changé le mobilier sans l'en prévenir ("La soustraction", 107). Dans "Zap" l'espace personnel devient même étonnamment perméable aux intrusions des autres à distance. Le héros de cette nouvelle vit seul et ne parvient même pas à simplement regarder la télévision tranquille chez lui puisque la télécommande des voisins vient interférer dans ses émissions pour lui en imposer d'indésirables. Il a beau essayer de se défendre en faisant zapper leur téléviseur à son tour, il sera le perdant de cette guerre quand les voisins trouvent le moyen de lui imposer par télécommande la vue d'un film porno où figure son exfemme, histoire d'avoir le dernier mot sur lui en le vexant d'une manière définitive.

Dans une autre nouvelle de Bergeron pourtant intitulée "Le refuge", significativement il n'y a pas de tranquillité possible. Le héros a choisi dans un hôtel calme 
hors saison une chambre en retrait dans une aile désertée. Seul sur son etage, il entend pourtant chaque soir un enfant longuement pleurer sans qu'on sache en localiser la provenance. L'espace intime est violenté de l'extérieur par le son, la souffrance des autres, les agressions diverses. Il n'y a jamais de repos, comme si le héros masculin n'avait pas droit à son espace privé.

A ces corrosions s'ajoutent les crises de couples qui souvent s'accompagnent de charivaris spatiaux. Dans "Méfiez-vous des cartomanciennes", toujours de Bergeron, le héros est un ex-policier devenu photographe qui connaît un divorce difficile et se voit victime d'une machination diabolique de la part de son ex-femme qui s'est associee à des complices pour le salir, lui faire perdre sa licence et l'obliger à quitter sa ville. Le combat est nettement territorial. En son absence on pénètre chez lui par effraction pour y déposer, il le découvrira, des photos de mineurs compromettantes auxquelles il n'a rien à voir. Alertés par un appel anonyme ses anciens collègues policiers, qui ont des comptes à régler avec lui parce qu'il avait mis au jour certains de leurs scandales, forcent leur entrée dans les lieux, brutalisent son occupant et perquisitionnent son domicile sans mandat. On voit d'ailleurs encore comment le monde public du travail et ses conflits viennent empoisonner l'espace privé masculin. Heureusement le héros aura été assez rusé pour comprendre vite les rouages pourtant compliqués de cette manigance et s'en sortira en retournant le vicieux procédé contre ses agresseurs. Ici l'espace privé de l'homme est attaqué, bafoué, trafiqué. On le détourne à des fins malhonnêtes, on l'envahit, on le saccage d'une manière criminelle. Aucune paix n'est possible.

Chez Girard on trouve aussi une crise analogue du privé liée au couple. Sa nouvelle kafkaïenne "La penderie" qui est extrêmement riche pour une topocritique en rapport avec la sexuation, est vraiment révélatrice d'une impossibilité territoriale du privé au masculin. Ici un homme qui vit seul à l'étroit dans une chambre est, en une inversion des stéréotypes, objet d'un voyeurisme permanent de la part d'une femme qui se cache et vit dans une penderie. Voila bien un dispositif spatial qui semble courant dans la littérature québécoise actuelle par lequel un homme se trouve sous l'observation d'une femme. Rien n'échappe à cette dernière de la vie personnelle de cet homme chez lui, de ses activités érotiques (solitaires et incongrues ou avec une autre femme), de son hygiène, de son sommeil, de la visite mystérieuse qu'il reçoit. Toute la narration est racontée du point de vue féminin. Si elle sait tout de sa vie privée, elle ignore en revanche tout de sa vie publique quand il sort de chez lui, mais elle en profite alors pour quitter sa penderie, assurer ses besoins vitaux (grappiller dans le frigo, par exemple) et surtout fouiller dans les affaires de l'homme sans que rien n'y paraisse. "Cette chambre devient mienne pendant ses courtes absences", dit-elle, "tout ici m'appartient" (145). Ce texte nous révèle entre autres le statut problématique de l'espace privé au masculin, tel que la nouvelle québécoise contemporaine le donne à percevoir. L'homme en est dépossédé et n'y a tout simplement pas droit pour lui-même.

Cette histoire est également éclairante sur le rapport que les deux sexes, quand ils partagent un même territoire, entretiennent avec l'espace privé. L'homme ignore tout de la

\section{LittéRéalité 66}


présence de cette voyeuse qui tient à sa discrétion absolue. Elle se dit pourtant amoureuse de lui, avoir besoin de son retour quand il sort. Elle vit dans la passivité, l'immobilité, le silence, la clandestinité, l'improductivité, la pauvreté d'un espace extrêmement réduit où elle se contente de vraiment peu et où ses propres affaires sont refoulées au fond d'une étagère. Certes, on peut y voir une sorte de transposition poussée jusqu'à l'absurde de la situation traditionnelle du couple, l'homme occupant la place dominante, passant du privé au public et la femme étant confinée au privé, au surplus soumise, effacée, inexistante, voire n'ayant plus qu'un rôle objectal similaire à celui d'un vêtement rangé dans un placard (sauf qu'elle n'assume aucune fonction domestique ici). Mais il y a plus, me semble-t-il, car s'y révèle en fait toute une conception de l'amour. Sa situation spatiale singulière lui plait, ce qui peut paraître incompréhensible à première vue: "j'y suis à mon aise", dit-elle (133). Les ténèbres de cette penderie sont des "ténèbres que j'aime" (142), elle en a besoin, ajoute-t-elle ensuite, pour "l'aimer et m'aimer" (142) sans "jamais lui parler" (144). "L'aimer et continuer de m'aimer. / C'est tout ce que je demande." (142). C'est qu'elle a une conception de l'amour qui est essentiellement fondée sur la séparation territoriale, l'isolement des corps, la noncommunication et un dépassement sensoriel qui confine à l'abstraction et au mysticisme. Elle rêve d'un amour au-dessus des sens. Voici comment elle décrit cet idéal amoureux en rapport avec l'espace: "Deux cœurs voisins habitant deux espaces contigus, sans issue visible, avec une fente dans la paroi mitoyenne, pour les lettres. Des chambres à ce point obscures qu'il sera impossible de s'y relire. Des lieux sans porte, avec une interstice dans le centre du mur commun, une fente très mince, les lèvres du mur..." (141) Ce qui serait un cauchemar absolu pour l'espace privé des personnages d'Aude devient ici un idéal féminin vu par un auteur masculin: l'espace intime de la vie à deux y est rêvé comme un monde cellulaire séparant les deux sexes, un monde sans sexualité, sans sensualité, sans rien en commun si ce n'est le mur qui les sépare l'un de l'autre et une mince fente de communication. L'intimité à deux adopte ainsi les caractéristiques traditionnelles du public: désincarnation, fonctionnalité, distance, abstraction, à quoi s'ajoute ce trait majeur reconnu à l'espace public masculin mais qui gagne aussi maintenant le privé: l'aliénation.

Girard est ici proche de la société future imaginée par Bergeron dans ses récits de science fiction ("Le monde aurait un nom" et "La soustraction"), où un "édit du mur" (117) opère une stricte ségrégation sexuelle qui va jusqu'à parquer les hommes et les femmes dans des espaces isolés et leur interdire de communiquer entre eux, chaque sexe devenant ainsi privé de la moitié de l'humanité. Mais Bergeron aime suivre les rebelles audacieux qui transgressent la loi du mur en traversant de l'autre côté. Comme quoi la passion quelle qu'elle soit peut toujours survivre aux reterritorialisations même les plus outrancières.

En conclusion, dans la très grande majorité des cas de notre échantillon l'espace privé ne bénéficie plus d'une pérennité qui l'installerait dans la solidité des choses rassurantes qui ne bougent jamais. Quand les deux sexes s'y retrouvent, soit il y a malaise entre eux par rapport à la situation d'intimité, soit ils connaissent un relatif bonheur mais il est limité, car cet espace privé se caractérise par une foncière fragilité. Quand il n'est pas miné de l'intérieur 
par quelque force négative, il est attaqué de l'extérieur. Chez l'homme surtout, l'espace intime n'est jamais respecté et se transforme en enfer, de sorte que le privé au masculin est ici vu comme une sorte de contradiction dans les termes. L'espace privé semble avoir perdu ses frontières étanches et protégées d'antan, il souffre d'impureté, de perméabilité, contaminé sans cesse qu'il est par l'espace public. En fait, le prive est en crise chez les deux sexes. Si chez la femme il résiste encore parfois aux assauts de l'extérieur, surtout chez les personnages d'Aude qui savent le remblayer dans certaines circonstances, si les deux sexes chez elle peuvent par moments apprivoiser un même espace et y vivre de rares interactions harmonieuses, l'espace personnel reste soumis à des secousses affectives qui lui sont fatales et ne résiste pas aux avatars de la finitude.

Au surplus les paramètres privé/public ne correspondent plus dans l'ensemble aux normes traditionnelles. Il y a un brouillage territorial dans la nouvelle québécoise contemporaine. Le public s'infiltre dans le privé, le privé sort dans l'espace public. Les conduites sont largement irrespectueuses des espaces qui ont pu leur être attribués dans le passé. Qui plus est, les classifications canoniques qui identifiaient le public au masculin et le privé au féminin sont remises en question. On peut donc déjà voir que l'espace de la nouvelle met le genre en crise.

\section{Ville/campagne}

Le monde urbain en opposition au rural: voilà un autre paramètre qu'abordent les nouvellistes québécois contemporains. Dans l'ensemble de la production, j'ai déjà pu noter que Montréal restait le point de rassemblement des effectifs du genre au cours de la dernière décennie, bien que les villes de province aient fait une entree non négligeable dans le paysage littéraire ${ }^{3}$.

La métropole est plus que jamais auparavant soumise à un regard critique où on en cadastre les coins sordides, où on la passe à quelque loupe sociologique pour en débusquer les petites misères, où on observe cette tristesse palpable "sur tous les balcons de la ville" qu'y voit Turcotte (62). Pour beaucoup Montréal devient la représentation même de la mélancolie (voir chez Monique Proulx ou Gilles Archambault, notamment).

Dans l'échantillon qui nous préoccupe seule Turcotte concentre l'essentiel de ses histoires à Montréal où elle habite. A l'exception d'un épisode de "Qu'y a-t-il à l'intérieur?" qui se déroule à Québec, ville de l'évasion et du désir, de l'espoir de renouveau et où son hérö̈ne fait la connaissance d'un amant pour lequel son cœur va palpiter un moment, Turcotte est résolument une nouvelliste métropolitaine dans "Caravane". Souvent la ville est explicitement identifié par un nom de rue (McGill College, Saint-Laurent, l'autoroute VilleMarie, la Place des Arts). En revanche la campagne ne l'intéresse pas comme nouvelliste. Elle évoque bien un motel au bord d'une grand route avec miroirs et films pornos réservés aux retrouvailles clandestines de son héroïne avec un de ses amants, mais c'est le plus loin de la ville où elle s'aventure.

A la différence des personnages de Turcotte, ceux d'Aude privilégient la campagne

\section{LittéRéalité 68}


sur la ville pour leurs entreprises d'épanouissement. La nouvelliste établit d'ailleurs une étanchéité territoriale entre l'espace urbain et le monde rural. "Le passeur" est très révélateur à cet égard. Son herroïne y tourne le dos au monde artificiel qu'elle a pu construire avec François à la ville et son décor de revue chic sans âme, avec tapis d'Orient, vaisselle de Limoges, meubles en palissandre, pour lui préférer l'espace de passion au sein de la nature que lui ouvre Carl, le passeur du titre. Si la ville est asexué, la campagne, elle, est sursexuée. Pour retrouver son amant, Delphine ici fraie son chemin à travers mûriers et joncs au bord d'un ruisseau où il l'attend. Ils s'aiment sur un lit de fougères, des épines lacèrent leur visage et leurs mains, des fruits laissent du sang sur leurs vêtements. Bref leur passion s'associe à l'univers végétal et aquatique de la nature. Le bonheur, la sensualité, la sexualité sont des épiphénomènes ruraux pour Aude. L'espace naturel loin de la ville n'est pas seulement libérateur, il est aussi salvateur. C'est ce qu'elle démontre encore dans "Les chiens" où un héros masculin vit seul sur une île d'un grand archipel où il fait l'inventaire des oiseaux et où ses guides sont deux chiens qui en savent plus que lui sur les lieux. Aude oppose nettement ici l'ancienne vie de son héros en usine où il s'est bagarré, d'où il a été congédié. En ville il a connu le chômage, les matinées à dormir, les après-midi à chercher un emploi, les soirées à s'écraser dans un sofa ou à boire avec des amis et à déprimer jusqu'à envisager le suicide. Maintenant sur son île, il fait corps avec la nature, les animaux lui apprennent le génie des lieux et il est plus heureux qu'à la ville.

Les nouvelles de Bergeron se passent occasionnellement à Montréal ou des épisodes y ont lieu, et alors limage qu'il en donne est plutôt négative (voir p. 83 par exemple). Avant tout il privilégie cependant une petite ville de province non identifiee dont on peut supposer qu'il s'agit de Thedford Mines où il vit. Mais si petite soit-elle, ce reste une ville que l'auteur tient à bien distinguer de la campagne, car pour lui celle-ci s'associe à la régression, à la dégradation et à la crise de couple. Dans "L'album de photos" un bref séjour à une maison de campagne conduit un homme et une femme à une scène de ménage et à leur brusque séparation sur une route déserte. Dans "L'œil tranchant" le fait de déménager de la ville à la campagne représente une réduction d'existence et une perte de qualité de vie. C'est, dit-il, se retrouver dans une "cabane de deux pièces" (46), dans un "coin perdu", loin de la famille, de la mer, dans des hauteurs où l'air se raréfie et où l'hiver n'en finit plus. Bref, le monde rural est à éviter autant que possible.

Quant à Girard il fait référence à Trois-Rivières, Joliette ou Hull autant sinon plus qu'à Montréal. D'ailleurs la métropole est tantôt un espace de compromis érotique où un héros de Charlesbourg a ses rendez-vous amoureux avec son amante de Hull, tantôt un espace de violence comme il le montre dans "A la merci", où son héros rencontre la mort à l'angle de la rue Sanguinet et Ontario. Girard est le seul de l'échantillon à exploiter les effets de brouillage autour des paramètres urbains et ruraux. Il le fait en employant des références campagnardes pour décrire la ville. Par exemple, les Montréalais y sont perçus comme des «bovins» et Trois-Rivières pue "le fumier de poule comme un lendemain de pluie, l'ensilage mal aéré" (18). D'une femme errant dans la ville, il dit qu' "elle sort, et marche dans les rues 
et les rangs sous le soleil et le crachin" (46). Le langage est complaisamment paysan, ce qui enracine le point de vue et donne un regard terrien sur les espaces bétonnés. C'est que pour lui la campagne ne diffère guère de la ville en ce que la bêtise, la violence, la déchéance y règnent tout autant.

Elle s'en démarque cependant sur le plan imaginaire en ce qu'il distingue deux sortes de campagne, l'une de la famille et du travail, l'autre, du loisir. Dans "La maîtresse de mon père" il oppose la perception d'un père à celle de son fils à propos du chalet familial. Pour le père qui s'y est réfugié, c'est un "enclave de liberté", alors que pour le fils, c'est une "chiotte déglinguée". La cuisine d'été où le père entraîne son fils pour lui faire la confidence stupéfiante qu'il a une mâtresse, lui apparaît "remarquaaable" (sic), tandis que pour l'autre elle n'est que "moisie et exiguë". Comme le point de vue narratif est celui du fils, c'est la vision de ce dernier qui finit par l'emporter et le dialogisme du texte abdique vite devant son témoignage accablant au profit d'un espace en ruines, laissé à l'abandon par le père, où le toit fuit et où on doit même porter des bottes à l'intérieur. La campagne ne peut pas être un lieu de détente pour Girard, car c'est fondamentalement un lieu de labeur. Le père est ici moins coupable de vivre à la campagne que d'être paresseux et négligent, donc inapte à la vie laborieuse que suppose implicitement la ruralité.

La vraie campagne du loisir existe pourtant chez Girard, mais elle se situera plus radicalement au nord, vers le haut, là où la nature est encore intacte. Cette campagne sauvage est un espace sportif, celui de la chasse et de la pêche, espace nettement masculin, mais où la présence féminine est maintenue à travers la nature, car une fois là-bas on pense, dit-il, aux "femmes, les délaissées en bas, celles-là (...) qui nous accompagnent à chaque pas dans chaque swamp..." (64). Il est significatif de voir que le nord est le pôle du désir chez Girard, celui du loisir sexuel. Aussi dans "La Femme-Subaru" la voiture follement érotique déjà évoquée roule-t-elle sans cesse de plaisir vers le nord. Les personnages de Girard n'hésitent pas à orienter leur vie en fonction de ce point cardinal du désir, car c'est aussi aux yeux de l'auteur un espace mythique de plénitude.

En conclusion, le genre sexuel ne sert guère de différenciateur dans la perception du paramètre ville/campagne chez les nouvellistes québécois contemporains. Le traitement en varie plutôt avec le lieu individuel d'ancrage de l'expérience et de l'écriture: il est déterminé par les avatars biographiques (selon que les auteurs vivent à Montréal, à Québec ou en province) plus que par une construction générique et notre échantillon ne permet pas d'associer à ce paramètre une sexuation particulière. Chez Aude l'épanouissement du couple a lieu à la campagne là où précisément il se défait chez Bergeron qui lui préfère la civilisation. On ne peut cependant pas en conclure pour autant que la femme a une propension rurale et l'homme, urbaine, car Turcotte, chez qui la sexualité du couple est surtout métropolitaine, ignore le monde rural alors que Girard, tout en brouillant les territoires, repousse la campagne vers l'univers sauvage du nord et y polarise idéalement le désir. Ainsi ce paramètre permet de tracer une cartographie rigoureusement mixte de la nouvelle et de mettre au jour une diversité géographique plus grande qu'on ne le dit

\section{LittéRéalité $\mathbf{7 0}$}


généralement.

\section{Fermeture/ouverture}

Aude est assurément la championne de la révolte contre la thématique de l'enfermement et de l'étouffement qu'on a pu associer à l'écriture féminine. A cet égard "Vases communicants" et "Tout est ici", nouvelles déjà évoquées, sont éclairantes de nouveau.

Dans "Vases communicants", nous voyons une héroïne alitée et claustrée dans une sorte de petit mouroir à treize lits. L'espace est occupé par deux groupes opposés, les intacts et les atteints (88). La narratrice y décrit sa descente aux enfers dans une atmosphère où les patients des deux sexes indifféremment rassemblés, la différence sexuelle ne servant plus à rien dans un tel monde et se trouvant frappée d'une dérisoire frivolité, s'unissent dans un râle commun. Voilà encore un espace oppressant où le rêve reste le seul refuge. Il n'y a aucun autre avenir envisagé que celui qui consiste à se voir passer du statut de patient à celui de mourant, ce qui se traduit en termes spatiaux par le déplacement du lit vers un petit salon d'où on ne sort qu'avec une étiquette mortuaire au gros orteil. Cette nouvelle nous présente donc la version féminine de la plus grande fermeture, celle de la finitude.

"Tout est ici" en montre le pendant masculin. Le héros narrateur y mène une vie horizontale dans un asile d'aliénés. L'espace est fermé. Il s'y sent prisonnier, y est maltraité: Il n'y a aucun réconfort à attendre des autres patients qui ne se parlent même pas, au contraire il y a tout à craindre d'eux, car ils se volent entre eux. Rien à espérer du personnel dit "soignant" non plus, qui ne se manifeste que par la violence: par exemple, on frappe le héros dès son arrivée parce qu'il vomit, de manière à ce qu'il comprenne qu'il n'est pas dans un lieu compatissant où on va prendre soin de lui. Cet espace est globalement bruyant et exhale une forte odeur de cadavre pourri. C'est litteralement l'enfer. La seule occupation qui les tienne consiste a jouer aux cartes avec des cartons qu'ils ont fabriqués et qui sont porteurs de désirs, ces cartes faisant référence à la nourriture, à la sexualité, à la propreté, à l'alcool, à l'exotisme, à l'humour, à la musique ou à l'art, bref à ce qui leur manque dans ce monde insalubre et aliénant où tout est torture. Les gagnants de ce jeu regagnent leur lit le soir avec au moins quelques rêves sur cartes dans la main, car c'est tout ce qui leur reste. Aude fait ici, de façon féroce, le procès des institutions psychiatriques en adoptant le point de vue de l'insoutenable, celui du patient. Comme pour "Vases communicants" c'est un espace étanche au monde extérieur et complètement inhumain. Pour homme ou pour femme, la fin est donc la même. Devant la mort il n'y a plus de distinctions genériques, il n'y a que des consciences vives enfermées dans des corps périssables.

Au-delà de ces fermetures négatives liées à la maladie et à la mort, Aude étudie tout particulièrement les origines psychologiques de l'enfermement. Au premier rang, elle en démontre l'enracinement familial. Elle consacre une nouvelle à ce thème, "L'envol du faucon", où elle évoque une fillette éduquée par une mère très sévère qui l'étouffe dans des vêtements trop serrés au point qu'elle développe un corps filiforme. L'enfermement provient 
encore d'une solitude brutalement imposée par la vie et qui conduit à la dépression entraînant dans son affaissement une transformation perceptive de l'espace. Dans "Cet imperceptible mouvement", l'héroïne en deuil de l'aimé n'a plus le même rapport à la maison de campagne qu'elle partageait avec lui. Elle y aperçoit des lézardes qu'elle n'avait jamais remarquées, s'imagine que les fondations se sont mises à bouger sous la structure qui va bientôt craquer comme une noix et glisser de la falaise dans l'eau (110), évite certaines pièces, ne peut plus ouvrir sans vomir les tiroirs du défunt ni le placard où ses vêtements sont encore suspendus au-dessus de ses chaussures alignées. La perception de l'espace est profondément altérée sous l'effet de l'émotion. Se trouvent inscrits dans le lieu de vie tous les contrecoups psychiques de ceux qui y habitent.

Chez Aude l'espace est ainsi toujours chargé d'affectivité, positive ou négative, et nous touchons ici au registre, important pour une topologie imaginaire, de ce que j'appellerais les pathotopes, où l'imagination présente les lieux en tant que porteurs de pathos: une maison de campagne est plus qu'un simple espace physique pour Aude, c'est un espace émotif qui se construit à travers le ressenti subjectif. Ici le moulin de la mélancolie est parvenu à broyer tous les signès autour du sujet pour les réduire en une poussière de tristesse une répandue sur tout et qui décolore tout l'espace. En outre dans ses représentations de pathotopes, Aude est particulièrement sensible à ce que je nommerais encore la transspatialité, c'est-à-dire les transformations subjectives successives d'un lieu sous l'effet d'un traumatisme. Ici naguère encore source de bonheur infini, cette maison de campagne n'est plus maintenant pour la survivante qu'une source intarissable de chagrin. Aude aime ainsi mettre en parallèle deux perceptions opposées d'un même pathotope: l'un, hypo-espace de départ, est perçu à un moment comme rempli de joie et l'autre, hyper-espace d'arrivee, devenu plus tard submergé de douleur affective, de sorte que tout en restant le même espace physique il n'a plus les mêmes résonances chez le sujet qui l'occupe.

Le pire enfermement est celui qui nous enferme en soi et pour Aude l'action la plus sûre c'est encore de s'ouvrir à l'autre et d'aimer à nouveau. Il suffit d'écumer les métaphores spatiales du "Passeur" pour le comprendre. Avant l'amour Delphine est "un bunker où elle s'est enterrée et dont elle ne trouve plus l'issue". Son nouvel amant, Carl, est "un obus qui fonce vers elle" et va la libérer de cette prison. Comme bien des personnages de nouvellistes actuels, l'amour naissant de Delphine et Carl passe par un sas de transit privilégié: une chambre d'hôtel minable, que l'héroïne évalue très positivement, en termes de vérité et d'authenticité: "Ici pas de quatuor à cordes pour masquer le vide ni de paravents chinois derrière lesquels se dissimuler. Il y a cette chambre sordide, sa lumière trop crue et cet homme en elle." (33) Ce lieu dépouillé devient un espace érotisé où l'homme fait craquer l'épaisse couche de laque qui recouvrait Delphine. Ils se retrouveront à la fin dans le nouvel appartement presque vide de l'héroïne où ils recommencent tout à neuf, dans un espace symbolique de renouveau qui, cette fois, se fondera sur le désir plus que sur les conventions. L'enfermement cesse chez Aude dès la rencontre de l'autre.

En contraste cependant avec Aude, chez Turcotte l'étouffement naît de la

\section{LittéRéalité 72}


confrontation à l'autre sexe, car la vie harmonieuse à deux dans un même espace y est impossible. Aussi dès qu'elle éprouve la moindre impression d'enfermement dans un lieu avec un homme, son hérö̈ne le quitte-t-il énergiquement. Elle démontre une grande aisance à claquer la porte d'une manière définitive sur un espace affectif. C'est le sujet de la nouvelle "Conversation" notamment. Le refuge dans la solitude lui est davantage tolérable, voire régénérateur car il ménage un retrait fécond, partie d'un espace privé qu'elle se construit, où le repli creuse un lieu de liberté en soi (113) et où, à travers soi, elle peut connaitre le monde. L'enfermement aliénant n'est pas son fait.

Dans un lieu fermé elle aimera certains micro espaces privilégiés d'ouverture vers l'extérieur, tel que la fenêtre, ouverture sensible et cognitive où nombre d'événements surviennent d'une manière enrichissante. Des drames de couples éclatent dans son cadre, des états d'âmes liés au climat en transpirent, des portraits d'autrui s'y révèlent. Un chez soi clos s'ouvre ainsi d'une manière enrichissante sur le dehors grâce à cette ouverture que représente une simple fenêtre. Turcotte recherche encore dans un lieu fermé des micro espaces d'ouverture vers soi, tel le divan, micro espace de la solitude réflexive où le repliement est gestation, fût de désirs, approfondissement, agrandissement de soi. Dans "Qu'y a-t-il à l'intérieur?" le divan prend une valeur particulière. "Sur le divan, chaque soir, je sentais qu'un espace s'ouvrait à l'intérieur de moi. Je changeais. " (113) Dans ce cas un rapport différent à l'espace familier peut apparaître: "Je suis devenue étrangère dans ma propre chambre." (114) Des remises en question peuvent s'ensuivre ainsi que des décisions de changement. Ici à la suite de sa rumination solitaire sur son canapé l'héroüne décide de s'ouvrir à l'amour un moment et, très significativement, elle décrit la liaison dans laquelle elle se lance en termes d'ouverture spatiale: "un passage s'ouvrait enfin", dit-elle (123), "j'ouvrais l'amour pour voir ce qu'il y avait à l'intérieur" (138). Turcotte, il faut dire, excelle à l'exploitation poétique de telles métaphores pathotopiques, elle qui aime tant pousser son héroïne à franchir "la frontière de son désir" (84), comme elle dit en une image spatiale libératrice. Chez Turcotte il y a ainsi une alternance entre un sentiment d'enfermement à deux et une solitude refermée mais germinative et libératrice qui ménage bientôt une ouverture sur une nouvelle liaison.

Même s'il arrive que la solitude soit douloureuse chez Turcotte elle est décrite comme la conséquence d'une décision personnelle qui la rend plus acceptable. L'origine en est une crise de couple, mais la résolution ultime, elle, est le fruit d'un choix. Dans "L'avenir de ma solitude" une photo non familière trouvée par hasard par la narratrice dans une pile de photos familiales, photo compromettante où l'on voit une inconnue sur une route déserte dans un paysage nordique, suffit à dévoiler une infidélité et entraînera la rupture du couple. L'héroïne commente ainsi sa décision de rompre: "Il fallait que je me perde, qu'il y ait un espace géographique inoui autour de moi, que cet espace soit le drame et que je sois comprise dans ce drame, mais d'une façon détachée, comme la femme sur la photo." (157) Elle cherchera à traduire sa solitude retrouvée en termes d'isolement géographique. Cette solitude se vit comme une fermeture sur soi dans un lieu ouvert. En s'imaginant faire corps avec le paysage, l'émotion féminine devient spatiale. Si une telle spatialisation du registre

Brulotte * 73 
affectif est commune à Aude et Turcotte, le traitement du paramètre fermeture, on le voit, est loin d'être univoque du côté féminin.

Dans l'imaginaire masculin de la nouvelie les signes spatiaux de la fermeture existent tout autant et ont une ambivalence analogue. Les espaces y sont souvent isolés, restreints, piégés ou traqués, habités par une solitude angoissée, mais leurs héros soit se débattent et s'en sortent (comme dans "Méfiez-vous des cartomanciennes" de Bergeron), soit s'accommodent de ces lieux (comme pour la narratrice de "La penderie" chez Girard ou pour le propriétaire de ce chalet sordide dans "La maîtresse de mon père"). A vrai dire certains personnages masculins et féminins chez eux souhaiteraient même plutôt pouvoir bénéficier d'un espace fermé, mais tranquille, étanche aux influences du dehors, qui leur servirait de refuge, ce qu'ils éprouvent beaucoup de mal à obtenir. Certains cependant y réussissent. Dans "L'écriture de la nuit", Bergeron présente une femme qui parvient à se réfugier dans ses rêves nocturnes, espace de retrait en soi où elle se construit une vie parallèle qu'elle ne partage pas avec son compagnon de vie. Les auteurs masculins sont aussi sensibles au thème de l'autoenfermement négatif. Bergeron l'etudie avec minutie et montre que ce thème touche autant les hommes que les femmes. Dans une nouvelle émouvante, "Le téléphone", une secrétaire de direction attend un appel qui ne viendra pas et tourne en rond. Elle va et vient entre le séjour où se situe le téléphone, la cuisine où elle renouvelle sans cesse son verre de vin et la salle de bains où elle prend comprimés sur comprimés. La seule issue à son drame personnel, puisque l'autre ne se manifeste pas, semble être le glissement dans l'abîme et le suicide. Chez ce nouvelliste l'enfermement vient surtout de la pauvreté intérieure des êtres, à moins qu'il ne soit imposé de l'extérieur comme il l'imagine à travers le thème de la ségrégation homosexiste qui hante ses récits de science fiction, ce qui achève de montrer combien l'enfermement menace les deux genres à égalité.

Une nouvelle de Bergeron, "Les fenêtres", bien que complexe dans sa brièveté (cet auteur est toujours exigeant pour ses lecteurs qui sont appelés à participer à la construction de l'histoire), me parait éclairante sur le rapport au paramètre de la fermeture et de l'ouverture. Le héros narrateur est seul à sa fenêtre et distingue différentes façons d'être en relation avec le dehors par l'entremise de cette ouverture. Il dit en avoir connu deux dans sa propre évolution. Dans le passé: "Je tenais à vivre dans un intérieur clair", où les fenêtres, essentiellement pourvoyeuses de lumière, servaient ses entreprises de lutte contre l'assombrissement. "A présent, argumente-t-il, les fenêtres, c'est différent. Je regarde dehors." "La lumière qui entre m'indiffere (...) elle le fait chez tout le monde. Moi je regarde à la fenêtre (...) Il y a sans cesse quelque chose qui bouge." (101) Surtout elle l'ouvre aux bruits. "Elle apprend à entendre." (102) Elle est mobilisation active des sens et communion avec l'extérieur. Par opposition, une jeune femme qui vit seule en face, ouvre son rideau chaque soir à heure fixe et se tient immobile à sa fenêtre. "Elle ne regarde nulle part", déduit le narrateur, "sinon droit devant elle, un regard inoccupé." (102) En plus de ne rien voir, le narrateur assure qu'elle n'entend rien autour d'elle. "Rien de ce que pourrait lui apprendre une fenêtre ne lui est accessible." (103) En fait, on apprend bientôt qu'elle se déshabille dans cet

\section{LittéRéalité 74}


encradrement et c'estdonc à un strip-tease que le narrateur assiste, spectacle qu'il décrit avec une impassible indifférence. Après s'y être déshabilleee, commente le narrateur: "Elle reste là en pure perte, comme paralysée, interdite" (103). Autrement dit, voici donc, selon le héros de Bergeron, la fenêtre féminine par opposition à la masculine. Dans l'une, la femme apparemment offre sa nudité en spectacle. Dans quel but? Est-ce pour séduire le voisin ou est-ce pour cruellement le tantaliser? Est-ce pour lui ou pour quelqu'un d'autre? Est-ce seulement pour quelqu'un? Est-ce par simple distraction et sans intentions érotiques particulières, par quelque mécanique gestuelle de l'être fatigué de sa journée? Est-ce un rite personnel avant de se mettre au lit? On ne saurait le dire, le texte maintenant une savante ambiguilté autour du geste. Toujours est-il que l'homme, lui, ne comprend pas l'offre, si offre il y a, ou ne la reçoit pas comme telle ou fait semblant de ne pas comprendre. Il juge la femme selon d'autres critères, exclusivement rationnels, dans sa relation à la fenêtre. Il croit avoir un rapport sensuel à ce micro espace d'ouverture, alors qu'il est en réalité dans l'abstraction. Il pense que la femme est abstraite, alors que c'est elle qui est sensuelle. Il la décrit comme passive et immobile, mais il l'est autant qu'elle. Ce à quoi la distinction tient cependant c'est que la femme ici ne s'ouvre pas sur l'extérieur de la même manière que lui: il la perçoit comme ayant une relation soumise à quelque retour narcissique sur soi comme si sa fenêtre était un miroir, alors qu'il se saisit lui-même dans la dynamique opposée, dedans-dehors, s'appropriant le monde extérieur de la fenêtre. En d'autres mots, il la croit dans l'enfermement et se voit dans l'ouverture. Mais en réalité les deux sont enfermés chacun chez soi, en soi, chacun dans sa propre solitude, dans la pauvreté affective et l'incapacité de communiquer. L'enfermement touche vraiment les deux genres.

Pour Girard aussi la fermeture est tantôt positive si elle est choisie (comme dans le repliement créateur), tantôt négative si elle est imposée. Mais l'enfermement aliénant ne l'intéresse guère dans Espaces à occuper et en cela il s'apparente à Turcotte tout en appuyant implicitement les dénonciations d'Aude. Sans cesse ses personnages préfèrent s'évader dans des lieux publics, on l'a vu, ou prendre la route en quête d'aventures. Leurs efforts ne sont pas toujours récompensés et la déception les attend souvent en bout de piste, mais il suffit d'une belle rencontre, une seule, pour que lêtre parvienne enfin à s'illuminer de tous ses feux. C'est bien ce qui arrive dans la dernière nouvelle du recueil, "La maîtresse de mon père", position stratégique s'il en est, où l'amour fou du héros pour une Française se cristallise à ciel ouvert sur les quais entre le Pont Neuf et le Pont Mirabeau et sème une pagaille surréaliste dans la cartographie de la Ville Lumière. Alors il recourt à une orgie de métaphores pathotopiques dynamiques pour dire ce qu'il éprouve. A bord du "long subway humide" de sa passion, il mélange les repères spatiaux, voit le marché aux puces aux Champs Elysées, la Tour Eiffel fichée dans le béton par son antenne (167), avant de se perdre dans "le nowhere" des caresses de sa maîtresse. En outre à l'instar de Turcotte, le sujet girardien spatialise son être émotif et aime voir l'amour en termes d'espace libre prêt à l'accueil de l'autre: "cette immense plaine, en moi, ce pourtant si petit lieu pour l'amour, nul ne pourra l'occuper comme elle." (169)

En conclusion, la fermeture n'est plus une thématique monovalente ni exclusivement

Brulotte 75 
féminine. Il y a une fermeture négative, qui est enfermement aliénant et une fermeture positive, qui est retrait régénérateur et épanouissant. L'univers masculin est préoccupé et affecté tout autant que le féminin par l'enfermement étouffant. La différence sexuelle n'importe plus face à l'incarcération définitive que représente la mort. Les femmes et les hommes se révoltent de l'enfermement, en étudient les causes psychologiques, y réagissent. En bout de piste pas d'enfermement qui tienne face à l'action, laquelle se traduit souvent par une ouverture renouvelée à l'autre par l'amour, car la pauvreté affective conduit à un repliement malheureux sur soi. Implicitement ce qui se dit ici d'une nouvelle à l'autre, c'est qu'on envisage, pour l'homme autant que pour la femme, l'ouverture amoureuse comme le remède universel à toute entreprise, effective ou potentielle, de fermeture.

\section{Mobilité/immobilité}

Quant au paramètre mobilité/immobilité, les quatre nouvellistes ne le campent pas non plus dans des positions imaginaires rigides. Girard, le grand agité de la nouvelle québecoise contemporaine, et pour qui suivant Poe qu'il cite en épigraphe "tout mouvement, de quelque nature qu'il soit, est créateur", conçoit tout autant que "limmobilité elle-même traduise une certaine amplitude du désir" (52). Au premier chef cependant c'est la mobilité qui le fascine et ses héros ne s'enferment guère dans la léthargie. Qu'importe qu'il y ait des ruptures, de la solitude ou des blessures affectives dans leurs entreprises puisqu'elles entrainent l'action et la transformation créatrice: "C'est le vide entraîné par l'absence qui permet -mieux: provoque- le mouvement" (69). Chez Girard la voiture, on l'a vu, sert de véhicule privilégié à la grande mobilité du désir chez les deux sexes. La Femme-Subaru chasse l'aventure en roulant à travers la ville ou sur les routes. Elle est de ces femmes désirantes à faire dresser les hommes sur une roue. A son image ses personnages masculins aimeraient devenir des Hommes-Subaru que l'héroine de Turcotte rêverait sans doute de rencontrer, puisqu'elle accorde la même confiance à la voiture pour ses vertus résolutives. Déjà dès son enfance, comme elle l'évoque dans "Friperie", ce véhicule constituait un moyen de fuite du domicile familial quand la vie y devenait tendue, car alors son père l'emmenait faire un "tour d'auto", comme elle dit, et lui offrait des glaces (18). Chez elle, la voiture ne sert pas que de nacelle aux amours voltigeantes, elle dessert les entreprises d'évasion et les quêtes de bonheur.

Bergeron et Aude s'en remettent également au mouvement pour régler les plus graves problèmes de l'existence, mais aussi pour atteindre une certaine forme d'épanouissement. Chez Aude, il sert à sortir de la dépression, car c'est l'action qui conduit un être à s'extirper de la passivité et qui seule le détermine vers un accomplissement autre. Dans "Cet imperceptible mouvement" la scur de l'héroïne vient la soutenir dans une telle reprise de soi après un deuil accablant. Ensemble elles finissent par travailler la terre et planter des fleurs, gestes simples mais qui suffisent à rebrancher la souffrante sur la vie. Dans "Méfiez-vous des cartomanciennes" de Bergeron, l'homme se tire du piège qu'on lui a tendu en traversant la ville souterraine en tous sens pour confondre ses poursuivants et en allant 
courageusement confronter son ex-femme.

Cependant bouger comporte aussi des risques. Nombre de nouvelles insistent justement sur les périls de la mobilité et en montrent ainsi l'ambivalence. Dans "Le monde aurait un nom" le héros de Bergeron prend l'initiative imprudente, dans le contexte, de rendre régulièrement visite à son aimée Rachel dans limmeuble des femmes, ce qui bafoue le grand interdit de la société futuriste qu'on nous présente ici et où règne un homosexisme rigide qui empêche hommes et femmes de se fréquenter. La route qu'emprunte encore cette "élue" dérisoire qu'est cette poule dans la fable girardienne ne conduit qu'à la mort prématurée et le pauvre animal finit comme un petit paquet d'os émiettés sur le pavé après avoir été écrasé par les voitures et mangé par un corbeau. Dans "L'effigie oubliée" de Girard à nouveau la voiture ne conduit guère plus loin qu'à l'urgence (94) et chez Bergeron des vacances à Kennebunk Port mènent toute une famille dans un précipice de trente mètres au bout d'un pont effondré ("Les vacances"), alors que dans "Iris" l'héroïne d'Aude a perdu ses parents en voyage, quand un pont de cordages suspendus s'est rompu sous leurs pieds au-dessus d'un gouffre (52). Lorsqu'elle n'engendre pas de catastrophes, la mobilité crée des situations instables (voir l'errance malheureuse chez Aude dans "L'enfant prodigue"), brise des liens intimes (provoque des divorces, par exemple, comme dans "Le colis de Kyoto" où un père a quitté sa famille québécoise pour aller vivre au Japon) et déchire le tissu social à la base. Chez Bergeron une certaine forme négative de mobilité masculine renvoie aussi au flottement de l'être en dérive qui n'arrive plus à s'ancrer dans le réel où il se sent devenir, ditil, "un élément mobile d'un décor sans formes", avec au cœur une nauséeuse impression "d'étale dans le temps, d'interchangeable dans les lieux" (17).

Le type de mobilité favori des nouvellistes québécois reste cependant le voyage, lequel contrebalance les risques associés au mouvement par des bénéfices considérables. J'ai déjà signalé combien la nouvelle de la dernière décennie a intégré le dépaysement par le voyage d'une manière sans précédent ${ }^{4}$. Les quatre auteurs de notre échantillon exploitent eux aussi cette thématique. Le voyage, les migrations, le mouvement en général forcent à repenser des notions comme celles d'identité et de subjectivité en déstabilisant le binarisme Occident/Orient et Nord/Sud hérité des Lumières et en développant des espaces de l'entredeux. Les échanges et la circulation des idées ont fait que la division entre l'étranger et le monde naguère clos des Québécois s'est estompée et que nombre de personnages de couleur, de religion et de nationalité différentes ont maintenant gagné le paysage culturel. Les nouvellistes actuels participent à ce phénomène migratoire. Ils ne voyagent pas qu'en touristes pour s'évader. Souvent les héros et les hérö̈nes connaissent ailleurs de vives expériences d'altérité, expériences qui les modifient en profondeur. Dans "Ultimo puerto" d'Aude, Xavier fuit vers le Sud dans un pays latino-américain où, après un mois de soûlerie transitoire qui le conduit au bord de la déchéance dans la clochardise, il découvre une femme patiente qui deviendra son phare. Sa vie s'en voit transformé de bout en bout. Il ne sera jamais plus le même. On a beau bouger et s'en aller au loin, on ne réussira à se libérer que si l'on ne reste pas enfermé dans ses problèmes, que si l'on s'ouvre réellement à l'autre. 
Chez Turcotte le voyage suscite de rares moments de plénitude, bonheur non pas de s'étourdir banalement dans le tourisme mais plus simplement d'être là dans une chambre de motel d'une station balnéaire de Nouvelle Angleterre à regarder un film avec les enfants pendant que le père de la narratrice, figure rassurante qui complète l'image familiale, est dans la chambre d'à côté. Ce moment est décrit comme "un pur ravissement" ("Lettres à personne") et conclut le recueil. Voilà un excellent exemple d'une femme qui donne une image positive de la relation père-fille, phénomène qui est rare dans la littérature québécoise moderne.

Chez Girard le voyage mène toujours à la découverte de l'amour vrai, que ce soit à Paris ou plus modestement dans une chambre d'hôtel ordinaire rue Saint-Hubert à Montreal où des amants provinciaux se retrouvent tous les mois depuis seize ans et sont heureux de ces moments de grande intensité qu'ils ont su maintenir dans la duree ("Amants"). Ils ont chacun leur vie, leur conjoint, leurs enfants, leur travail, mais rien ne remplace ces retrouvailles clandestines loin de chez eux où rires et larmes se mélangent au plaisir. De temps en temps quand ils écartent les jalousies de la fenêtre ce n'est pas pour s'extasier devant plages et palmiers, mais pour regarder la lune ou le chantier d'en face à la gare routière. Seul un abandon provisoire de son territoire qui n'a rien à voir avec l'exotisme permet de telles expériences heureuses de l'altérité.

Et que dire encore des immigrés, de ceux qui viennent d'ailleurs et aident les indigènes à réévaluer leur propre vécu local. Ils se sont déplacés de là-bas vers ici pour trouver une vie meilleure, une vie comme celle que les indigènes mènent et qu'ils ne savent pas toujours apprécier. La nouvelle québécoise a accueilli un grand nombre de personnages immigrés au cours de la dernière décennie. Elle est devenue résolument interculturelle et, ce faisant, aborde les nouveaux problèmes que cette situation crée, ainsi que je l'ai analysé ailleurs ${ }^{5}$. Girard nous le rappelle quand dảns "Soirée barattée" son héros rencontre une Noire venue des pays chauds, attirée qu'elle était par les contrées glaciales et qui laisse la fenêtre ouverte en hiver, confirmant ainsi la polarisation nordique du désir de son univers.

Le voyage n'est pas que spatial, c'est entendu, il est aussi temporel et nous touchons alors au vaste thème de la chronotopie qui mériterait un long développement à lui seul. Avant tout les personnages aiment se déplacer dans le passé. Dans une émouvante nouvelle sur l'enfance "Portrait de nous devant la vitrine", la Marie de Turcotte chérit des moments de son passé associés à des activités et à des lieux précis: à la boutique horlogère de son père, où elle se rappelle sa fascination de le voir réparer des montres; à la rue Saint-Laurent, où elle a connu la joie de faire du lèche-vitrines en compagnie d'une fillette et de ses parents, d'entrer avec eux dans les magasins de vêtements pour enfants; à un café des alentours où elle a éprouvé un instant de plénitude autour d'un gâteau au fromage et d'un verre de lait. A l'occasion de tels chronotopes simples où l'imagination a le sens du temps dans l'espace, tout semble s'intégrer pour produire la merveille d'un espace-temps comblé, arraché à la grisaille générale de la vie. "Je ne pouvais rien désirer de plus", dit-elle (67). "J'étais à l'intérieur de l'amour". Moment emboîté, encoconné, protégé. "La tristesse n'arrivait plus jusqu'à moi."

\section{LittéRéalité 78}


Mais le chronotope peut aussi, bien sûr, être porteur de souffrance. C'est ce que vit encore Marie dans une "Friperie" où elle retrouve, fâcheux hasard, sa propre mère, une mère détestée qui reprend aussitôt le pouvoir sur elle et choisit les vêtements à sa place. Cette rencontre lui fait revivre une humiliation maternelle subie dans son enfance a l'occasion d'un essayage dans un lieu similaire. Au sein de ce chronotope Marie vit donc en abrégé le phénomène de mémoire involontaire par lequel cette "friperie" quitte son plat statut d'espace commercial pour devenir un espace-temps rempli d'ulcérations personnelles venues du passé, ingérence d'une intimité indésirable dans le public.

Les nouvellistes sont aussi sensibles aux marques que peuvent laisser dans l'espace ces autres dimensions temporelles que sont la saison et le moment du jour, ainsi qu'à leur incidence sur la mobilité ou l'immobilité des personnages. Par exemple dans "Le paradis de tous les possibles" de Turcotte, l'hiver, la neige, le grand froid rendent Marie malade, l'obligeant à la vie immobile et ralentie chez soi. L'immobilité a donc son temps saisonnier, comme elle a son lieu. On voit alors l'espace de l'héroïne se laisser infléchir sous la pression hivernale et se rétrécir. Son parcours dans son intérieur où elle est confinée devient très limité: de sa chambre à son bureau, de son bureau à la cuisine, parcours qui s'alourdit de "toutes sortes de choses intimes" qu'on entraîne avec soi (79).

Girard et Aude ont en commun une chronotopie qui aime présenter un espace aux prises avec le temps et modifié par lui tout en renvoyant à la dynamique du sujet. Dans "La maîtresse de mon père" le héros de Girard insiste sur la condition de délabrement extrême du chalet de ses parents, laissé délibérément en l'état: or, le père ne voyage pas et préfere envoyer son fils à Paris pour qu'il rencontre sa maîtresse à sa place. Ici l'espace est à la foisgrugé par le travail destructeur du temps et par l'inertie patemelle, une inertie qui révolte le fils et qui se paiera cher, car le fils voué, lui, sans cesse à la mobilité du désir lui volera sa maîtresse. Dans "Période Camille" d'Aude, Thomas, artiste peintre, vient d'acheter un manoir où a vécu sa sœur avec un homme qu'il n'appréciait pas. Jadis opulent, ce lieu est désormais à l'abandon. Lorsqu'il était occupé par les amants tout y était dominé et dompté: jardin géométrique, roseraie, etc... Maintenant que l'amour n'y habite plus, la bâtisse s'effrite, la peinture des pergolas et des tonnelles s'écaille, les sentiers sont couverts de mousse, de lierre, de plantes mortes, de mauvaises herbes, et tout se fond peu à peu à la forêt environnante. L'espace soumis à la corrosion temporelle participe aussi de cette transspatialité typique d'Aude qui montre combien un lieu, sous les secousses affectives de ses occupants, peut évoluer dans le temps. Il n'est point besoin de s'agiter physiquement pour bouger, car ce qu'Aude appelle "cet imperceptible mouvement" de l'espace et du temps, bien que fort lent, suffit a indiquer la dynamique subjective en jeu. Un espace dont la vocation affective change est un espace voué à la déhiscence et à la destruction. Désertés par l'amour ces espaces ne peuvent plus revivre comme avant. C'est sans doute en partie ce qu'Aude veut signifier quand elle dit que, dans ses toiles, Thomas finit par brouiller les lieux de Camille qu'il cherchait à dessiner et par les éclabousser de couleurs. 
De notre échantillon, c'est Aude qui offre la chronotopie la plus riche. Pour elle l'espace actuel sert toujours d'essor à la mémoire. Dans "Cet imperceptible mouvement" l'héroïne s'assoit dans une balançoire de bois qui lui rappelle aussitôt celle de la cour familiale d'antan ainsi qu'une scène d'enfance (115). A travers la verte transparence des yeux d'Iris, dans la nouvelle qui porte ce prénom floral, Alexandre revoit l'eau de cette île, tout au sud, où il avait failli se noyer huit ans auparavant (61). Un souvenir spatial apparaît en palimpseste sur une perception présente et ici vient étayer la fascination éprouvée pour l'autre. Une telle mobilité imaginaire, signe de vitalité, continue même avec la maladie. Dans les récits hospitaliers d'Aude l'espace se voit davantage saturé de temps, un temps dramatisé et tragique, à la fois lent, presque immobile, et compté, qui porte la fatalité en lui et conduit en raccourci à la mort. Le temps humain s'y trouve ramassé dans un petit espace réduit, étouffant, d'où il n'y a aucun échappatoire, mais il reste les rêves, si dérisoires qu'ils soient, par lesquels une moribonde voyage un peu en prenant la Rivière des Prairies pour la Seine.

En conclusion la mobilité, tous genres confondus, offre elle aussi une polyvalence de fond avec ses versants positifs et négatifs pour les deux genres. On ne vote pas les yeux fermés pour elle contre son contraire, puisque la mobilité comporte des dangęrs bien réels. Cependant une des formes favorites de cette mobilité reste le voyage que les deux sexes effectuent pour vivre des expériences d'altérité et de réconfort qui les transforment décisivement. Il y a aussi le voyage immobile, temporel celui-là, et les nouvellistes élaborent des chronotopies riches où la poésie rivalise avec la profondeur psychologique et philosophique. Dans l'ensemble, nous assistons pour ce paramètre à une déstabilisation analogue à celle que nous avons observée ailleurs, la mobilité n'ayant plus une polarité masculine ni l'immobilité, une féminine.

\section{Les entre-deux symboliques}

A en croire les nouvellistes retenus les quelques refuges qui restent relèvent d'un imaginaire de l'entre-deux qui redéfinit les rapports à deux. Pour Bergeron les espaces sacrés (églises, cathédrales) sont des lieux à la fois publics et intimes, tout de tranquillité, de cérémonies rituelles, de stabilité, de prévisibilité, de conservation. Un de ses héros y est heureux, non pas tant pour l'expérience religieuse que pour la paix. "Il est si rare de se trouver en des lieux privés d'événements", dit-il d'une église dans "La soustraction" (109), endroit dont le décor ne change pas, où les paroles ne connaissent pas de modifications. "Entre ces murs, conclut-il, on se sent justifié d'être le même." (106) Mais significativement ce sont des espaces asexués où l'autre sexe n'a pas de place.

Au contraire pour Aude le lieu sacré est un espace d'étouffement parce que lié au célibat et à cette infamie que représente, pour elle, la séparation des sexes. Un de ses héros, dans "La nuit obscure", est un religieux qu'elle saisit alors que sa foi bascule, soudainement rongée par le désir sexuel. Il croyait vivre dans la paix et la lumière au fond de son abbaye, mais brusquement après des années de retraite, troublé par sa belle-sœur, tout lui devient insupportable. Il se sent en exil dans son lit étroit, l'air lui est si lourd dans la serre qu'il croit

\section{LittéRéalité 80}


respirer de l'eau, la puanteur de la fromagerie l'indispose, les bruits domestiques l'exaspèrent (le cliquetis des cuillères dans les bols, les bruits de succion et de déglutition dans le silence glacé du réfectoire), les couloirs sinistrement résonnent comme dans une maison vide, il ne parvient plus à poursuivre le plain-chant avec ses confrères. C'est qu'il vient d'accéder à la vraie lumière, profane et sensuelle celle-là, ce qui le fait passer de l'ascèse à la passion. La nature asexuée de l'espace mystique n'a ainsi rien pour plaire à Aude. Quand on s'y trouve, le mieux c'est d'en sortir au plus vite car il n'y a pas d'issues de ce côté. Pour elle l'espace heureux est, par définition, nécessairement hétérosexualisé.

Les réflexions philosophiques et mystiques viendront plutôt à ses personnages non pas de l'espace carcéral des cellules monastiques, mais de l'espace ouvert du cosmos. Le silence infini du monde sidéral murmure une réponse plus authentique au questionnement métaphysique et incite à une humilité quasi religieuse. Dans "Cet imperceptible mouvement", elle intègre une telle scène méditative à un chronotope de plénitude quand Justine se revoit avec Pierre transportant leur lit dehors à l'occasion de la nuit des Perséides pour contempler les étoiles filantes. Alors la voie lactée fait découvrir au couple le caractère périssable de tout. "Rien n'est immortel" conclut-on (114) de cette expérience. Ici le cosmos est un espace à la fois ouvert et fermé, un univers d'expansion et de retour sur soi qui ménage bien des compromis imaginaires et soulève des réflexions majeures.

Parmi les refuges de l'entre-deux, il y a l'art. Pour Turcotte la musique semble pouvoir accomplir des miracles, elle qui remodèle l'espace et innerve tout l'être. En taxi dans la nuit de "Comment vivre le soir de son anniversaire", accompagnée de sa dernière conquête, elle donne au chauffeur une cassette à faire jouer. Aussitôt la bande sonore construit une bulle invisible autour d'elle. "La musique était l'endroit rêvé pour mon âme", dit-elle (74). C'est encore la musique qui l'attire dans tel lieu public, ou qui l'envoûte et la transporte dans tel autre. Grâce à cet art, elle réussit à conjoindre un besoin de solitude avec la nécessité d'une présence aimée.

Chez Bergeron l'art a un statut ambigu, car c'est un intermédiaire à la fois exaltant et inquiétant entre les êtres. Dans "Mazin Taïno" il est si fascinant qu'il engendre des expériences suprasensibles: dans un musée, le héros entend la voix intérieure d'une figure féminine représentée sur une toile. L'œuvre d'art agrandit les capacités du moi et devient médiatrice du désir en lui faisant rencontrer une femme énigmatique, qui est sans doute celle du tableau, car sa présence auprès de lui chaque midi au restaurant du musée coïncide avec la progressive disparition de la figure picturale des cimaises, comme si l'imaginaire se répandait dans le réel. En soi ce pourrait être merveilleux que l'art serve ainsi à satisfaire des désirs, mais chez Bergeron il a aussi le pouvoir excessif, irrationnel et dangereux d'emporter le spectateur dans l'irréel, de le soustraire à la vie pour l'annexer à l'univers artificiel du tableau. Dans "L'œil tranchant", il décrit une toile qui hypnotise, immobilise, rend prisonnier et même esclave, voire vampirise et provoque un état comateux qui prépare le sujet à sa réification esthétique. "Je suis devenu la chose de ce tableau, un morceau d'un bloc posté devant cette toile..." (40) La perception de l'art ne conduit pas au calme, mais au contraire 
à quelque troublante dépersonnalisation ou incite à devenir autre que soi. De là à croire que l'art est meurtrier comme le pensait un Thomas de Quincey quand il évoquait "l'assassinat par les Beaux-Arts", il n'y a qu'un pas que le nouvelliste franchit. Pour lui l'art peut en effet comporter un aspect fascisant et en imposer à celui ou à celle qui le fréquente, au point de susciter des comportements indésirables entre les sexes. Cet effet semble universel tant il en relève des cas analogues ailleurs, à Paris où un tableau a poussé une femme à dépecer son compagnon de vie, ainsi qu'à Buenos Aires, où une peinture sert de canalisations à des frustrations de couples et pousse encore une fois une femme au meurtre de son partenaire. Donc réserve et prudence s'imposent au contact de l'art: certes, il agrandit le moi et suscite des rencontres inédites, mais son magnétisme est quelque peu redoutable...

Quant à Aude, pour elle l'art peut constituer un refuge, mais à la condition qu'il se nourrisse de l'autre. Il n'est pas la cause d'une relation, mais sa résultante. On le voit aux deux espaces artistiques qu'elle présente dans son recueil: le loft d'Alexandre dans "Iris", qui est aussi son studio de photographe et le manoir de Thomas dans "Période Camille". Dans les deux cas, c'est grâce à l'amour plus qu'à l'art que la chambre noire du photographe cesse d'être noire et que le manoir traverse ses heures de gloire. L'amour a le pouvoir pour Aude, si sensible aux pathotopes, de remodeler l'espace et sans amour tout ne serait que chambre noire ou manoir en ruines. Pour que l'art soit un ciment viable entre les êtres, il a besoin de l'amour qui le subsume.

Quant à l'art littéraire, les nouvellistes ne cessent de l'évoquer dans les quatre recueils. C'est significativement à son secrétaire, son unique refuge ultime, que l'homme traqué de "La Penderie" peut se retirer à la fin. C'est dans le recueillement de l'écriture qu'il règle une partie de sa solitude, c'est-à-dire son versant stérile, mais pour atteindre cet état de grâce, il enferme involontairement sa voyeuse parasite dans le placard en poussant une malle contre la porte. Dans un texte inclassable " Manuel d'abandon? de carrière? 20 leçons simples " Girard invite, en une mise en abyme soutenue de l'écriture, à refuser certaines conventions sociales et à oser sa vie du côté de la création, seul espace de salut où toutes les contradictions se résolvent. Chez Turcotte le pupitre de son héroïne l'accueille et la réconforte aux moments difficiles en lui permettant de s'évader dans l'imaginaire et d'écrire des histoires qui donnent une forme à son chagrin en la disposant vers un autrui rêvé. Quant à Aude "Fleurs de pavot" montre un écrivain qui redécouvre le plaisir d'écrire dans un espace partagé avec sa compagne artiste. L'art soude l'homme et la femme parce qu'ils s'aiment et l'écriture ne peut s'épanouir que dans la proximité de l'autre, voire qu'en s'accompagnant étroitement du désir sexuel. Chez Bergeron on doit se méfier de l'écriture comme de l'art, car c'est un refuge fragile puisque même l'écriture la plus privée qui soit, celle du journal intime, n'est pas protégée des intrusions extérieures. Dans la nouvelle éponyme "Visa pour le réel" une diariste s'aperçoit que son compagnon lit son journal en secret. Elle décide en réaction d'inventer des épisodes pour le rendre jaloux. Le journal perd les deux traits principaux qui le caractérisent: son intimité et son authenticité. On l'écrit désormais pour l'autre et il devient artifice. L'écriture n'est pas un refuge sûr, pas plus que l'art, puisqu'elle rend vulnérable aux

\section{LittéRéalité 82}


indiscrétions.

En conclusion sur l'imaginaire de l'entre-deux et les rapports entre les sexes qu'il redéfinit, on va de la quête désespérée de refuges sacrés asexués chez Bergeron où l'on peut goûter aux vertus de l'ascédie, à leur rejet catégorique chez Aude qui leur préfère la méga cloche de la voie lactée sous laquelle le couple peut se brancher sur les pulsations de l'univers et se nourrir de contemplation. Le refuge artistique, tout comme celui de l'écriture, a ses adeptes et ses réconforts chez les deux sexes, mais surtout ses conditions d'exercice qui varient de l'un à l'autre: solitude requise et résolutive des conflits chez Girard, fragile et génératrice d'angoisses face à l'autre chez Bergeron, refusée chez Aude, intermittente chez Turcotte. Chez les sujets masculins, l'écriture est salut pour le premier sous condition d'une hétérogénéité territoriale exclusive de l'autre, mais objet de méfiance pour le second parce que nécessairement dépendante de la présence implicite de l'autre ce qui expose à la victimisation. L'écriture comme refuge ne fait donc pas l'unanimité du côté des hommes. Chez les sujets féminins la perception du refuge créateur est également partagée: Turcotte l'associe à l'évasion, à un art de soi qui esthétise la vie émotive et qui, dans son exercice, se contente d'interactions sporadiques avec l'autre, alors que chez Aude l'activité créatrice est partie d'un monde sensuel tout entier habité par l'autre, et ne pouvant s'exercer qu'avec l'autre, l'amour étant, plus que tout art, l'ultime refuge d'où origine le sens. On voit ainsi que les solutions spatio-symboliques divergent non pas tellement d'un sexe à l'autre mais plus pertinemment d'un ou d'une nouvelliste à l'autre. Plus que génériques, elles sont rigoureusement individuelles.

\section{Conclusion générale}

Ainsi dans les quatre recueils parcourus, Cet Imperceptible mouvement d'Aude, Visa pour le réel de Bertrand Bergeron, Espaces à occuper de Jean Pierre Girard et Caravane d'Elise Turcotte, où domine l'hétérosexualité on constate que l'espace sert de critique aux distinctions génériques. Les paramètres spatiaux public/privé, ville/campagne, fermeture/ouverture, mobilité/immobilité perdent leur monolithisme et gagnent en polyvalence, en mixité. Les nouvellistes les déstabilisent en refusant ou en décloisonnant les classifications traditionnelles d'espaces masculins et d'espaces féminins spécialisés, tout en imaginant des reterritorialisations autres qui redefinissent les relations des sexes entre eux. Les conduites déployées et les émotions éprouvées par chacun des genres ne sont pas celles que la culture patriarcale a toujours réparties spatialement. En associant la femme et l'homme aux mêmes espaces il devient désormais difficile, voire impossible de demander lequel est l'espace de l'un, lequel, celui de l'autre. On peut en conclure que l'espace de la nouvelle met le sexe en crise.

Voilà donc ce qu'a à nous offrir ce genre innovateur sur le plan de l'espace et de la sexuation et j'espère avoir démontré que nous avons intérêt à écouter la force disruptive de cette fille rebelle du monde littéraire. Territoire réputé "mineur"au sein du grand domaine 
paternel de la littérature où domine le maître Roman, la nouvelle n'en pose pas moins des problèmes majeurs. Au terme de cette petite analyse j'aimerais souhaiter qu'un jour on se penche avec plus d'attention sur l'école québécoise de la nouvelle et qu'on mesure mieux sa profonde originalité au sein de la littérature mondiale. Si cette réflexion pouvait seulement contribuer à susciter cet intérêt, j'estime que j'aurai atteint mon but.

\section{Bibliographie}

\section{Oeuvres étudiées}

Aude. Cet Imperceptible mouvement. XYZ Éditeur, 1997, 119p

Bergeron, Bertrand. Visa pour le réel. Québec, LInstant même, 1993, 123p.

Girard, Jean-Pierre. Espaces à occuper. Québec, L'Instant même, 1993, 179p.

Turcotte, Élise. Caravane. Leméac, 1994, 171p.

\section{Études consultées}

Claridge, Laura et Elizabeth Langland (1990), Out of Bounds. Male Writers and Gender(ed) Criticism, Amherst, The University of Massachussetts Press.

Duncan, Nancy, dir. (1996), BodySpace. Destabilizing Geographies of Gender and Sexuality, New York, Routledge.

Sennett, Richard (1974), The Fall of Public Man, New York, Knoff.

Sinfield, Alan (1994), Cultural Politics -Queer Reading, Philadelphia, U. of Pennsylvania Press.

Threagold, Terry et Anne Cranny-Francis (1990), Feminine, masculine and Representation, Sydney, Allen \& Unwin.

\section{Notes}

${ }^{1}$ Voir mon article récent "Bilan de la nouvelle québécoise des dix dernières années du siècle." University of Toronto Quaterly 70.3, eté 2001: 768-799. Je me permets de renvoyer également à mes articles précédents sur le sujet: "Une Décennie de nouvelles québécoises: 1980-1990". The French Review [Etats-Unis] 65.6 Mai 1992: 963-977. "Formes de la nouvelle québécoise contemporaine". Ss dir. de Marcato-Falzoni, Franca et Lise Gauvin. L'Age de la prose. Romans et récits québécois des années 80. Montréal: VLB. Rome: Bulzoni, 1992. 67-84. Le présent texte est la version analytique d'une communication beaucoup plus brève faite à Groningen aux Pays-Bas dans le cadre d'un colloque international sur Espace et sexuation dans la littérature québécoise et dont les actes seront publiés en 2002 à Montréal aux Editions Nota Bene.

${ }^{2}$ Dans sa représentation de la mobilité la voiture est d'ailleurs si cruciale quil sent le besoin de nous identifier les marques favorites de ses personnages, de la Ford Ranger à l'Escort et 
même dintégrer une publicité sur la Subaru au corps de son texte, assurément une première stylistique dans la nouvelle québécoise.

${ }^{3}$ Je me permets de renvoyer à nouveau à mon article récent, "Bilan de la nouvelle québécoise des dix demières années du siècle." University of Toronto Quaterly 70.3, été 2001: 768-799.

${ }^{4}$ Art. cité.

${ }^{5}$ Loc. cit. 